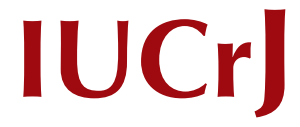

ISSN 2052-2525

CHEMISTRY|CRYSTENG

Keywords: chirality; structure analysis; configurational change; phase transitions; intermolecular interactions; amino acids.

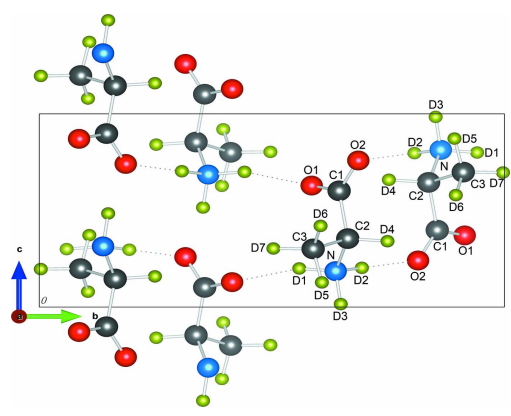

OPEN $\odot$ ACCESS

\section{Response to comment on 'Hydrogen bonds in crystalline D-alanine: diffraction and spectroscopic evidence for differences between enantiomers'}

\author{
Ezequiel A. Belo, ${ }^{\text {a,b }}$ Jose E. M. Pereira, ${ }^{\text {c }}$ Paulo T. C. Freire, ${ }^{b}$ Dimitri N. Argyriou, ${ }^{d}$ \\ Juergen Eckert ${ }^{\mathrm{e}, \mathrm{f}}$ and Heloisa N. Bordallo ${ }^{\mathrm{c}, \mathrm{d}_{*}}$
}

${ }^{\mathbf{a}}$ Faculdade de Física, Universidade Federal do Pará, Belém, Pará, Brazil, bepartamento de Física, Universidade Federal do Ceará, Fortaleza, Ceará, Brazil, 'Niels Bohr Institute, University of Copenhagen, Universitetsparken 5, Copenhagen, 2100, Denmark, ${ }^{\mathbf{d}}$ European Spallation Source, 176, SE-221 00 Lund, Sweden, ${ }^{\mathbf{e}}$ Department of Chemistry, University of South Florida, 4202 East Fowler Ave, Tampa, FL 33620, USA, and ${ }^{\mathrm{f}}$ Theoretical Division, Los Alamos National Laboratory, Los Alamos, NM 87545, USA. *Correspondence e-mail: bordallo@nbi.ku.dk

In the preceding comment on our paper Bürgi \& Macchi (2018) stated 'The recent paper by Belo, Pereira, Freire, Argyriou, Eckert \& Bordallo [(2018), IUCrJ, 5, 6-12] reports observations that may lead one to think of very strong and visible consequences of the parity-violation energy difference between enantiomers of a molecule, namely alanine' and 'Therefore, the conclusions drawn by Belo et al. (2018) are deemed inappropriate as the data presented do not contain sufficient information to reach such a conclusion'. In response to this comment we would like to stress the point that we did not in fact draw any conclusions at all in our paper concerning the parity-violating energy difference (PVED) hypothesis of Salam [Salam (1992), see also Laerdahl et al. (2000) and Berger \& Quack (2000) for discussion] and therefore find it difficult to see how they could therefore be 'deemed inappropriate'.

Belo et al. (2018) reports a careful parametric (temperature-dependence) study of D-alanine by polarized single-crystal Raman spectroscopy and neutron powder diffraction and makes comparisons with results on both $\mathrm{L}$ - and $\mathrm{D}$-alanine drawn from the literature. At temperatures where the structural information from the reported neutron powder diffraction measurements can be compared with previous single-crystal X-ray diffraction in L-alanine (Lehmann et al., 1972; Destro et al., 2008) and single-crystal neutron diffraction in L- and D-alanine (Wilson et al., 2005), there is good agreement when the difference between hydrogenated and deuterated samples is taken into account. The results reported by Belo et al. (2018), however, provide a continuous picture of the temperature evolution of the bonds in D-alanine from $280 \mathrm{~K}$ down to $4 \mathrm{~K}$, which shows that while the average structure is kept the same (no changes in space group) in D-alanine, as opposed to L-alanine, local symmetry changes are seen at lower temperatures. Furthermore, below $250 \mathrm{~K}$, both L-alanine and D-alanine appear to undergo microconformational transitions resulting from a subtle rearrangement of the hydrogen-bond network. This temperature corresponds with that where bulk measurements (Barthès et al., 2002, 2003; Wang et al., 2000, 2002; Sullivan et al., 2003) have observed anomalies that were indicative of a phase transition. Although it should be noted that Sullivan et al. (2003) were able to reduce this anomaly by re-growing their sample, it has been observed in a number of different samples prepared by different groups and should therefore be considered to be a real effect of as yet undetermined nature.

We note that the results of Belo et al. (2018) do not provide, or claim to provide, evidence for, or against, the Salam hypothesis, which predicted that quantum mechanical cooperative and condensation phenomena may give rise to a second-order phase transition below a critical temperature linking the transformation of $\mathrm{D}$-amino acids to L-amino acids. An order of magnitude estimate by Salam (1992) indicated a transition temperature of $\sim 250 \mathrm{~K}$. The work of Belo et al. (2018) does not support the idea of the D-alanine (D-ala) $\rightarrow$ L-alanine (L-ala) transformation, but instead provides a microscopic picture of the alanine solids consistent with the other experimental measurements. The properties of $\mathrm{L}$ - and $\mathrm{D}$-alanine, and the $\mathrm{L}$ - and $\mathrm{D}$-amino acids in general, are a fascinating, and important, area of study for our understanding of nature, irrespective of whether they are related, or not, to the weak nuclear force and parity violation. 


\section{scientific comment}

\section{Acknowledgements}

HNB would like to thank Mark Hagen from fruitful discussions.

\section{References}

Barthès, M., Dénoyer, F., Lorenzo, J.-E., Zaccaro, J., Robert, A., Zontone, F. \& Bordallo, H. N. (2003). Eur. Phys. J. B - Condens. Matter. 37, 375-382.

Barthès, M., Vik, A. F., Spire, A., Bordallo, H. N. \& Eckert, J. (2002). J. Phys. Chem. A, 106, 5230-5241.

Belo, E. A., Pereira, J. E. M., Freire, P. T. C., Argyriou, D. N., Eckert, J. \& Bordallo, H. N. (2018). IUCrJ, 5, 6-12.

Berger, R. \& Quack, M. (2000). ChemPhysChem, 1, 57-60.

Bürgi, H.-B. \& Macchi, P. (2018). IUCrJ, 5, 654-657.
Destro, R., Soave, R. \& Barzaghi, M. (2008). J. Phys. Chem. B, 112, 5163-5174.

Laerdahl, J. K., Wesendrup, R. \& Schwerdtfeger, P. (2000). ChemPhysChem, 1, 60-62.

Lehmann, M. S., Koetzle, T. F. \& Hamilton, W. C. (1972). J. Am. Chem. Soc. 94, 2657-2660.

Salam, A. (1992). Phys. Lett. B, 288, 153-160.

Sullivan, R., Pyda, M., Pak, J., Wunderlich, B., Thompson, J. R., Pagni, R., Pan, H., Barnes, C., Schwerdtfeger, P. \& Compton, R. (2003). J. Phys. Chem. A, 107, 6674-6680.

Wang, W., Min, W., Bai, F., Sun, L., Yi, F., Wang, Z., Yan, C., Ni, Y. \& Zhao, Z. (2002). Tetrahedron Asymmetry, 13, 2427-2432.

Wang, W., Yi, F., Ni, Y., Zhao, Z., Jin, X. \& Tang, Y. (2000). J. Biol. Phys. 26, 51-65.

Wilson, C. C., Myles, D., Ghosh, M., Johnson, L. N. \& Wang, W. (2005). New J. Chem. 29, 1318. 